\title{
Rising incidence of psychiatric disorders before diagnosis of immune-mediated inflammatory disease
}

\author{
R. A. Marrie ${ }^{1,2 *}$, R. Walld ${ }^{3}$, J. M. Bolton ${ }^{4}$, J. Sareen ${ }^{4}$, J. R. Walker ${ }^{5}$, S. B. Patten ${ }^{6}$, A. Singer ${ }^{7}$, L. M. Lix ${ }^{2}$, \\ C. A. Hitchon ${ }^{1}$, R. El-Gabalawy ${ }^{5,8}$, A. Katz ${ }^{2,3,7}$, J. D. Fisk ${ }^{9}$ and C. N. Bernstein ${ }^{1}$, for the CIHR Team in \\ Defining the Burden and Managing the Effects of Psychiatric Comorbidity in Chronic \\ Immunoinflammatory Disease \\ ${ }^{1}$ Department of Internal Medicine, Max Rady College of Medicine, Rady Faculty of Health Sciences, University of Manitoba, Winnipeg, Canada \\ 2 Department of Community Health Sciences, Max Rady College of Medicine, Rady Faculty of Health Sciences, University of Manitoba, Winnipeg, \\ Canada \\ ${ }^{3}$ Manitoba Centre for Health Policy, Max Rady College of Medicine, Rady Faculty of Health Sciences, University of Manitoba, Winnipeg, Canada \\ ${ }^{4}$ Department of Psychiatry, Max Rady College of Medicine, Rady Faculty of Health Sciences, University of Manitoba, Winnipeg, Canada \\ ${ }^{5}$ Department of Clinical Health Psychology, Max Rady College of Medicine, Rady Faculty of Health Sciences, University of Manitoba, Winnipeg, \\ Canada \\ ${ }^{6}$ Department of Community Health Sciences, Cumming School of Medicine, University of Calgary, Calgary, Canada \\ ${ }^{7}$ Department of Family Medicine, Max Rady College of Medicine, Rady Faculty of Health Sciences, University of Manitoba, Winnipeg, Canada \\ ${ }^{8}$ Department of Anesthesia and Perioperative Medicine, Max Rady College of Medicine, Rady Faculty of Health Sciences, University of Manitoba, \\ Winnipeg, Canada \\ ${ }^{9}$ Departments of Psychiatry, Psychology \& Neuroscience, and Medicine, Dalhousie University, Halifax, Canada
}

Aims. After the diagnosis of immune-mediated inflammatory diseases (IMID) such as inflammatory bowel disease (IBD), multiple sclerosis (MS) and rheumatoid arthritis (RA), the incidence of psychiatric comorbidity is increased relative to the general population. We aimed to determine whether the incidence of psychiatric disorders is increased in the 5 years before the diagnosis of IMID as compared with the general population.

Methods. Using population-based administrative health data from the Canadian province of Manitoba, we identified all persons with incident IBD, MS and RA between 1989 and 2012, and cohorts from the general population matched 5 : 1 on year of birth, sex and region to each disease cohort. We identified members of these groups with at least 5 years of residency before and after the IMID diagnosis date. We applied validated algorithms for depression, anxiety disorders, bipolar disorder, schizophrenia, and any psychiatric disorder to determine the annual incidence of these conditions in the 5-year periods before and after the diagnosis year.

Results. We identified 12141 incident cases of IMID (3766 IBD, 2190 MS, 6350 RA) and 65424 matched individuals. As early as 5 years before diagnosis, the incidence of depression [incidence rate ratio (IRR) 1.54; 95\% CI 1.30-1.84) and anxiety disorders (IRR 1.30; 95\% CI 1.12-1.51) were elevated in the IMID cohort as compared with the matched cohort. Similar results were obtained for each of the IBD, MS and RA cohorts. The incidence of bipolar disorder was elevated beginning 3 years before IMID diagnosis (IRR 1.63; 95\% CI 1.10-2.40).

Conclusion. The incidence of psychiatric comorbidity is elevated in the IMID population as compared with a matched population as early as 5 years before diagnosis. Future studies should elucidate whether this reflects shared risk factors for psychiatric disorders and IMID, a shared final common inflammatory pathway or other aetiology.

Received 29 June 2017; Accepted 18 September 2017; First published online 3 November 2017

Key words: Cohort, epidemiology, inflammatory disease, psychiatry.

\section{Introduction}

Various chronic conditions are characterised by immune dysregulation and inflammation that results in acute exacerbations and often progressive disability.

* Address for correspondence: R. A. Marrie, Health Sciences Center, GF-543, 820 Sherbrook Street, Winnipeg, MB R3A 1R9, Canada.

(Email: rmarrie@hsc.mb.ca)
Among these immune-mediated inflammatory diseases (IMID), inflammatory bowel disease (IBD), multiple sclerosis (MS) and rheumatoid arthritis (RA) share several epidemiologic and clinical features (Pugliatti et al. 2002; Health Canada, 2003; Alamanos et al. 2006; Canadian Institute for Health Information, 2007; Logan \& Bowlus, 2010; Bernstein et al. 2012). As compared with the general population, the incidence and prevalence of psychiatric comorbidity,

This is an Open Access article, distributed under the terms of the Creative Commons Attribution licence (http://creativecommons.org/licenses/by/ $4.0 /)$, which permits unrestricted re-use, distribution, and reproduction in any medium, provided the original work is properly cited. 
including depression, anxiety, bipolar disorder and schizophrenia, are increased in individuals with these IMID (Hauser et al. 2011; Matcham et al. 2013; Marrie et al. 2015b). This psychiatric comorbidity negatively affects outcomes; depression is associated with lower health-related quality of life in IBD, MS and RA (Rupp et al. 2004; Mitchell et al. 2005; Faust et al. 2012; Mok et al. 2012), and with increased mortality in MS and RA (Ang et al. 2005; Marrie et al. 2015a).

Prior studies suggest that the incidence of depression and anxiety may increase before the diagnosis of IMID. A Danish study of 5084 persons with MS and 24771 age- and sex-matched controls reported increased odds of a diagnosis of depression or anxiety in the 2 years before MS diagnosis but did not evaluate other psychiatric disorders (Hoang et al. 2016). In a study of 351 individuals with prevalent IBD that found an increased risk for mood and anxiety disorders, the onset of these psychiatric comorbidities most often predated the diagnosis of IBD (Walker et al. 2008). However, this study lacked a matched cohort from the general population for comparison.

The findings in IBD and MS suggest that psychiatric comorbidity may be the earliest manifestation of the onset of IMID in some individuals, and that common underlying aetiologies of psychiatric comorbidity may exist across IMID. Using population-based incident cohorts of three IBD, MS and RA, and matched cohorts from the general population, we aimed to estimate the incidence of several psychiatric disorders, including depression, anxiety, bipolar disorder and schizophrenia, in the 5-year periods pre- and post-IMID diagnosis. We hypothesised that the incidence of psychiatric comorbidity would be higher in the incident IMID than in the matched general population cohorts pre- and post-IMID diagnosis.

\section{Methods}

\section{Setting}

This retrospective cohort study was conducted in the province of Manitoba, Canada. Health care in Canada is a provincial responsibility, and is publicly funded for all residents. Records of health service delivery are prospectively captured electronically and are accessible for research. The University of Manitoba Health Research Ethics Board approved the study, and the Manitoba Health Information Privacy Committee approved data access.

\section{Data sources}

Since 1984, all Manitoba residents have a unique personal health identification number linked to health system contacts. We accessed de-identified administrative databases at the Manitoba Population Research Data Repository of the Manitoba Centre for Health Policy, including the population registry, hospital discharge abstract database (DAD), physician claims and Drug Program Information Network (DPIN). The population registry captures demographic information, dates of health care coverage and residence location as defined by postal code. The DAD captures all hospitalisations including admission and discharge dates, and up to 25 diagnoses. Until 2004, diagnoses were recorded using the International Classification of Disease Ninth revision, clinical modification (ICD)-9-CM codes, and have been recorded using the ICD Tenth revision, Canadian version (ICD-10-CA) thereafter. Physician claims capture the date of service and one diagnosis recorded using ICD-9-CM codes. The DPIN database, which dates from 1995, captures all outpatient prescription dispensations including the date, drug name and drug identification number, which is connected to the World Health Organisation's Anatomical Therapeutic Chemical (ATC) Classification System (WHO Collaborating Centre for Drug Statistics Methodology, 2012). We linked these databases using scrambled, unique provincial identifiers to ensure confidentiality.

\section{Study populations}

First, we applied validated case definitions (Bernstein et al. 1999; Marrie et al. 2010; Hitchon et al. 2014) to the administrative dataset to identify all Manitobans aged 18 years and older with IBD, MS and RA from 1984 to 2013 (Supplementary Table S1). For each condition, we assigned the date of diagnosis (i.e., index date) as the date of the first health claim for that condition. For each condition, we selected a cohort from the general population, matched $5: 1$ on sex, year of birth within \pm 5 years and region as defined by forward sortation area (first three digits of postal code). These matched cohorts excluded individuals with any diagnostic codes for IBD, demyelinating disease, RA or related disorders as well as anyone with prescriptions for MS-specific disease-modifying therapies (which contributed to the MS case definition) from 1984 to 2013.

Second, we selected incident cases of IBD, MS and RA (and their matched controls) by requiring a 5-year run-in period before the index date without any related claims. A small number of individuals met the case definitions for more than one IMID $(n=$ 165). We did not exclude them to ensure generalisability of our findings, but allowed them to count only once in analyses in which we created a combined IMID cohort. In this situation, they were classified on the basis of the IMID with the earliest index date in 
the coverage period. Third, we limited the analysis to incident cases and controls with at least 5.5 continuous years of data before and after the index date (spanning 11 years). Cases had index dates ranging from 1989 to 2008. We defined the 6-month periods on either side of the index date as the 'diagnosis year', the 5 years before the diagnosis year as the pre-diagnosis period and the 5 years after diagnosis as the post-diagnosis period.

\section{Psychiatric disorders}

Using case definitions developed and validated in MS and IBD populations (Marrie et al. 2013; Marrie et al. 2016), we identified individuals in each cohort with depression, anxiety disorder, bipolar disorder, schizophrenia and any psychiatric disorder ( $\geqslant 1$ of depression, anxiety disorder, bipolar disorder, schizophrenia) (Supplementary Table S1). For each psychiatric disorder, we assigned the diagnosis date as the date of first health claim for that disorder.

\section{Covariates}

Covariates included age at index date [grouped as 18-24 (reference), 25-44, 45-64, $\geqslant 65$ years], sex [female $v$. male (reference)], index year [1989-1998 (reference) v. 1999-2007], annual number of physician visits unrelated to psychiatric disorders, urban $v$. rural region of residence (urban regions included Winnipeg, population $>600000$ and Brandon, population $>47000$ ), and socioeconomic status (SES) at the index year in quintiles (highest quintile of SES as reference). For SES we used the Socioeconomic Factor Index version 2 (SEFI-2), derived by linking postal code to census data. The SEFI-2 combines information regarding average household income, per cent of singleparent households, unemployment rates and high school education rates; scores less than zero indicate better SES (Chateau et al. 2012). We also included the number of physician visits to account for possible surveillance bias due to increased health system contacts.

\section{Analyses}

Within each cohort (IBD, MS, RA and their controls), we estimated the incidence of each psychiatric disorder during the diagnosis year, and each year within the pre-diagnosis and post-diagnosis periods. We ageand sex-standardised incidence rates to the 2010 Canadian population, and compared them for each year separately using incidence rate ratios (IRR) and $95 \%$ confidence intervals $(95 \% \mathrm{CI})$ using a negative binomial distribution. We tested whether there were temporal trends in the incidence of psychiatric disorders within the pre- and post-diagnosis periods and whether these trends differed between the preand post-diagnosis periods. Findings between the IMID cohorts and matched controls were compared. We constructed multivariable negative binomial regression models that included main effects for case (case $v$. matches), period [pre-diagnosis (reference), diagnosis year, post-diagnosis] and year as a continuous variable $(1,2,3,4,5$ in each of the pre-diagnosis and post-diagnosis periods; 0 was the year of diagnosis), as well as two-way interaction terms between case and period, and between year and period, and a three-way interaction between case, year and period. These analyses were conducted in two ways: (ii) combining all IMID into a single 'case' group, and all matches into a single 'matched' group; and (iii) including a separate variable for each IMID and its matches in the same analysis. These models adjusted for all prespecified covariates. Given preliminary findings of an elevated incidence of psychiatric disorders as early as 5 years pre-diagnosis, we repeated the analysis, limiting it to incident cases and matches with at least 10.5 continuous years of data before and after the index date (spanning 21 years) to see if the findings were present as early as 10 years pre-diagnosis.

\section{Results}

\section{Study populations}

We identified 12141 incident IMID cases; 3766 had IBD, 2190 had MS and 6350 had RA. The combined matched controls included 65424 individuals. In the combined IMID cohort, most were females, living in urban settings at the index date (Table 1). Those in the MS and RA cohorts were more likely to be female than those in the IBD cohort. Those in the RA cohort had a later mean age at diagnosis than those in the IBD or MS cohorts.

\section{Incidence of psychiatric disorders in the combined IMID cohort}

In the combined IMID cohort, the age- and sexstandardised incidence of any psychiatric disorder rose during the pre-diagnosis period, peaked during the diagnosis year, then declined slightly postdiagnosis but remained higher than in the prediagnosis period (Fig. 1).

The age- and sex-standardised incidence of any psychiatric disorder among the IMID cohort was higher than the matched cohort during all periods, beginning as early as 5 years before IMID diagnosis (IRR 1.60; 95\% CI 1.36-1.89), and most prominently in the diagnosis year (Fig. 2, Table 2).

Similarly, the adjusted regression analyses showed that in the IMID cohort, the incidence of any 
Table 1. Characteristics of incident disease and matched cohorts at the index date

\begin{tabular}{|c|c|c|c|c|c|c|c|c|}
\hline Characteristic & $\begin{array}{l}\text { IBD matches } \\
(n=20355)\end{array}$ & $\begin{array}{c}\text { IBD } \\
(n=3766)\end{array}$ & $\begin{array}{l}\text { MS matches } \\
(n=12315)\end{array}$ & $\begin{array}{c}\text { MS } \\
(n=2190)\end{array}$ & $\begin{array}{l}\text { RA matches } \\
(n=33584)\end{array}$ & $\begin{array}{c}\text { RA } \\
(n=6350)\end{array}$ & $\begin{array}{c}\mathrm{IMID}^{\mathrm{a}} \\
(n=12141)\end{array}$ & $\begin{array}{l}\text { IMID matches } \\
\qquad(n=65424)\end{array}$ \\
\hline Female, $n(\%)$ & $11230(55.2)$ & $2062(54.8)$ & $9108(74.0)$ & $1621(74.0)$ & $24692(73.5)$ & $4658(73.3)$ & $8220(67.7)$ & 44427 (67.9) \\
\hline Age at diagnosis, mean (S.D.) & $41.0(15.9)$ & $41.5(16.4)$ & $40.5(11.7)$ & $40.2(11.5)$ & $52.3(15.3)$ & $52.7(15.4)$ & $47.0(16.2)$ & $46.6(16.0)$ \\
\hline $\begin{array}{l}\text { Duration of follow-up from the index } \\
\text { date (years), mean (s.D.) }\end{array}$ & $13.6(5.1)$ & $13.8(5.1)$ & $13.7(5.1)$ & $13.8(5.0)$ & $13.0(5.0)$ & $13.0(4.9)$ & $13.4(5.0)$ & $13.4(5.0)$ \\
\hline \multicolumn{9}{|l|}{ Region of residence, $n(\%)$} \\
\hline Urban & $13545(66.5)$ & $2491(66.1)$ & $8172(66.3)$ & $1435(65.5)$ & $19637(58.5)$ & $3681(58.0)$ & $7495(61.7)$ & $40809(62.4)$ \\
\hline Rural & $6810(33.4)$ & $1275(33.8)$ & $4143(33.6)$ & $755(34.5)$ & $13947(41.5)$ & $2669(42.0)$ & $4646(38.3)$ & $24615(37.6)$ \\
\hline Socioeconomic Factor Index score, mean (S.D.) & $-0.20(0.89)$ & $-0.28(0.92)$ & $-0.20(0.87)$ & $-0.27(0.90)$ & $0.08(1.00)$ & $0.04(1.00)$ & $-0.11(0.99)$ & $-0.06(0.96)$ \\
\hline
\end{tabular}

IBD, inflammatory bowel disease; MS, multiple sclerosis; RA, rheumatoid arthritis; IMID, immune-mediated inflammatory disease, combining IBD, MS and RA cohorts; socioeconomic status, Socioeconomic Factor Index scores; values less than zero indicate higher socioeconomic status.

${ }^{\mathrm{a}} \mathrm{A}$ small number of individuals met the case definitions for more than one of the IMIDs of interest. We did not exclude them to ensure generalisability of our findings (since IMID can be comorbid with each other), but only allowed them to count once in the IMID cohort. In this case, they were classified on the basis of the IMID with the earliest index date in the coverage period. 

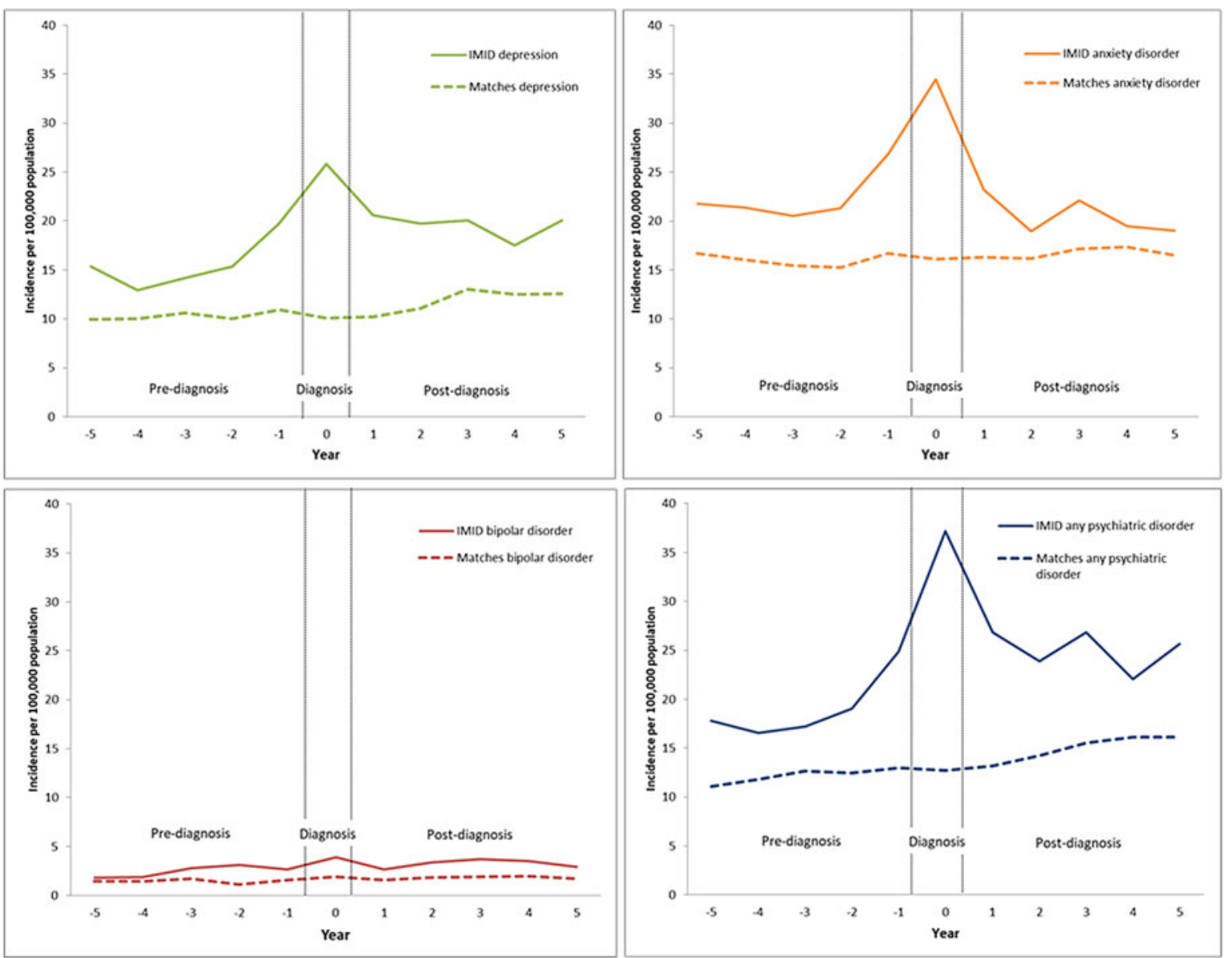

Fig. 1. Age- and sex-standardised incidence rates of psychiatric disorders in the immune-mediated inflammatory disease (IMID) and matched cohorts 5 years before and 5 years after IMID diagnosis.

psychiatric disorder rose in the pre-diagnosis period, and declined slightly during the post-diagnosis period (Table 3). In the matched cohort, the incidence of any psychiatric disorder rose slightly over time, but this pattern was the same in the pre- and post-diagnosis periods.

When we examined the incidence of depression, the findings were similar to those for any psychiatric

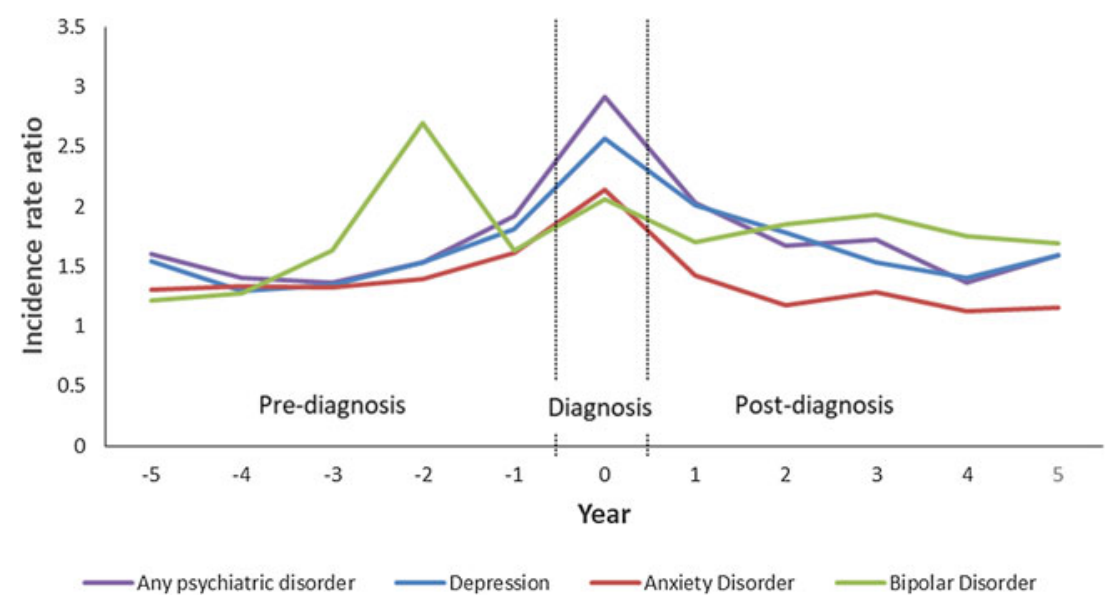

Fig. 2. Age- and sex-standardised incidence rate ratios for psychiatric disorders in the immune-mediated inflammatory disease (IMID) and matched cohorts 5 years before and 5 years after IMID diagnosis. 
Table 2. Incidence rate ratios (95\% confidence intervals) comparing age- and sex-standardised incidence of psychiatric disorders in the year of diagnosis ${ }^{a}$

\begin{tabular}{lcccr}
\hline & IBD & MS & RA & IMID \\
\hline Any psychiatric disorder & $3.11(2.44-3.95)$ & $4.66(3.03-7.17)$ & $2.52(1.85-4.34)$ & $2.92(2.56-3.32)$ \\
Depression & $2.76(2.10-3.62)$ & $3.62(2.49-5.27)$ & $2.25(1.57-3.23)$ & $2.57(2.21-2.99)$ \\
Anxiety disorder & $2.50(1.97-3.18)$ & $2.90(2.13-3.96)$ & $1.42(1.07-1.89)$ & $2.14(1.87-2.44)$ \\
Bipolar disorder & $1.73(0.93-3.23)$ & $3.29(1.78-6.08)$ & $1.38(0.82-2.33)$ & $1.88(1.35-2.62)$ \\
Schizophrenia & $\mathrm{b}$ & $\mathrm{b}$ & $\mathrm{b}$ & $1.71^{\mathrm{c}}(0.73-4.01)$ \\
& & & &
\end{tabular}

\footnotetext{
a Age- and sex-standardised to the 2010 Canadian population.

bSuppressed due to small numbers.

${ }^{c}$ Crude rate ratio as number of those affected too few to standardise incidence rates.
}

disorder (Fig. 1, Tables 2 and 3). For anxiety disorders, the pattern of the findings was similar to those for any psychiatric disorder and depression (Fig. 1). Although the incidence of anxiety disorder was higher in the IMID cohort than in the matched cohort, this was not statistically significant in the fourth and fifth years post-diagnosis (IRR 1.12; 95\% CI 0.94-1.34; IRR $1.15 ; 95 \%$ CI $0.96-1.38)$. While the incidence of anxiety was higher in the IMID cohort than in the matched cohort pre-diagnosis, the regression analyses did not demonstrate any change in the incidence of anxiety over the pre-diagnosis period (Table 3 ).

For bipolar disorder, we observed a similar pattern of findings, including an increased incidence in the IMID cohort relative to the matched cohort emerging 3 years before IMID diagnosis (IRR 1.63; 95\% CI 1.10-2.40) (Fig. 1, Table 2). Although the incidence of bipolar disorder rose during the pre-diagnosis period, this temporal change did not reach statistical significance (Table 3).

The incidence of schizophrenia in the IMID cohort was not statistically significantly higher in the diagnosis year than in the matched cohort either in the diagnosis year (Table 2), or pre-diagnosis. However, these findings should be viewed cautiously given the small number of individuals affected in each cohort; the small numbers also precluded regression analyses.

\section{Incidence of psychiatric disorders in the individual IMID cohorts}

In the analyses of each IMID cohorts (IBD, MS and $\mathrm{RA})$, the findings were generally consistent in magnitude and direction with those of the combined IMID cohort (Supplementary Table S2 and Figs S1-S3). In the diagnosis year, the age- and sex-standardised incidence of any psychiatric disorder, depression and anxiety disorder was higher in each IMID cohort as compared with its matched cohort (Table 2). In the IBD cohort, the incidence of any psychiatric disorder and depression were elevated relative to its matched cohort beginning 5 years pre-diagnosis, and rose over the pre-diagnosis period. The incidence of anxiety disorder was significantly elevated beginning 3 years pre-diagnosis. The incidence of any psychiatric disorder, depression and anxiety disorder were elevated beginning 5 years pre-diagnosis in the MS cohort $v$.

Table 3. Adjusted incidence rate ratios (95\% confidence intervals) for the association between immune-mediated inflammatory disease (IMID) and psychiatric disorders

\begin{tabular}{lllll}
\hline Variable & Any psychiatric disorder & Depression & Anxiety disorder & Bipolar disorder \\
\hline $\begin{array}{l}\text { Combined IMID cohort } \\
\quad \text { Year: cases pre-index }\end{array}$ & $1.08(1.03-1.13)$ & $1.07(1.02-1.12)$ & $1.03(0.98-1.07)$ & $1.11(0.99-1.24)$ \\
$\quad$ Year: cases post-index & $0.93(0.90-0.96)$ & $0.95(0.92-0.98)$ & $0.89(0.86-0.92)$ & $0.98(0.91-1.05)$ \\
Post-pre ratio: cases & $0.86(0.81-0.90)$ & $0.89(0.84-0.94)$ & $0.87(0.82-0.92)$ & $0.88(0.77-1.00)$ \\
Year: controls pre-index & $1.03(1.01-1.04)$ & $1.01(0.99-1.03)$ & $1.00(0.98-1.01)$ & $1.04(1.00-1.08)$ \\
Year: controls post-index & $1.04(1.03-1.06)$ & $1.04(1.02-1.06)$ & $1.01(0.98-1.03)$ & $1.01(0.97-1.05)$ \\
Post-pre ratio: controls & $1.02(0.99-1.05)$ & $1.03(1.00-1.07)$ & $1.02(0.99-1.04)$ & $0.98(0.91-1.05)$ \\
\hline
\end{tabular}

The year variable assesses whether there is an annual linear increase in incidence in the group (cases or controls) and period (pre-index or post-index) of interest. Post-pre ratio compares the year effect in the post-index $v$. pre-index periods. A ratio less than one indicates the yearly rise in incidence was greater in the pre-index than the post-index period. A ratio greater than one indicates the yearly rise in incidence was greater in the post-index than the pre-index period. 
its matched cohorts and rose over the pre-diagnosis period. In RA, the incidence of anxiety disorder was elevated as compared with the matched cohort at all times, but did not rise significantly over the prediagnosis period. In the IBD, MS and RA cohorts, the incidence of bipolar disorder was elevated as compared with the matched cohorts beginning to 2-3 years pre-diagnosis, but the rise in incidence over the pre-diagnosis period did not reach statistical significance (Supplementary Fig. S3). The data for schizophrenia could not be modelled due to small cell sizes.

\section{Sensitivity analyses}

When we restricted the analyses to members of the combined IMID and matched cohorts with 10 years of data before and after the diagnosis year, characteristics of the cohorts were similar to those of the original cohort (Supplementary Table S3), as were the findings in the diagnosis year (Supplementary Table S4). The pattern of findings regarding the incidence of the psychiatric disorders was also similar in that the increased incidence of any psychiatric disorder was already evident 10 years pre-diagnosis (IRR 1.50; 95\% CI 1.10-2.06) in the combined IMID cohort (Supplementary Fig. S4), and 8-10 years pre-diagnosis in the individual IMID cohorts (data not shown).

\section{Discussion}

Using population-based data and an observational design, we evaluated whether the incidence of psychiatric disorders increases before the initial diagnosis of IMID. Unlike previous studies, we studied three IMID simultaneously to identify common patterns, and we evaluated multiple psychiatric disorders. We focused on incidence rather than prevalence because this is more relevant for understanding the aetiology of disease, and provides greater clarity in understanding of the temporal associations between IMID and psychiatric disorders. As compared with age-, sexand geographically matched cohorts, the incidence of psychiatric comorbidity was increased in the IMID cohorts in the 5-10 years before IMID diagnosis. The similarity in findings across three IMID suggests a common underlying biology for psychiatric disorders in IMID.

Our findings are consistent with a limited number of prior studies that have suggested higher than expected incidence or prevalence of depression and anxiety before the diagnosis of IBD or MS (Kurina et al. 2001; Walker et al. 2008; Byatt et al. 2011; Hoang et al. 2016); we were unable to identify comparable studies for bipolar disorder. In one study of 187 individuals with IBD and a lifetime history of any mood or anxiety disorder, $18 \%$ had onset of these disorders within 2 years preceding IBD diagnosis and an additional $16 \%$ had onset 2-9 years before diagnosis (Walker et al. 2008). A study of the ulcerative colitis form of IBD that used physician claims data from one health region in Alberta, Canada found that neuroses/depression were more likely to occur before IBD diagnosis although psychotic disorders did not. A British case-control study using administrative data found that the prevalence of depression and anxiety was higher than expected in the year before diagnosis of ulcerative colitis, and the prevalence of depression was higher more than 5 years before diagnosis (Kurina et al. 2001). A Danish study using administrative data found that the odds of a diagnosis of depression or anxiety were higher in MS $v$. matched controls in the 2-year period before MS diagnosis (OR 1.4; 95\% CI 1.05-1.88) (Hoang et al. 2016). In an interview-based study of 29 individuals with MS, 17 had a lifetime prevalence of major depressive disorder, of whom four reported this as a prodrome preceding neurologic symptom onset (Byatt et al. 2011).

We found that the incidence of depression and anxiety disorder was increased substantially in the year of IMID diagnosis. The period surrounding the diagnosis of chronic illness can be associated with increased depressive symptoms that may or may not worsen over time (Chiu et al. 2016). One British study suggested that the risk of depression and anxiety were highest in the first year after diagnosis of IBD (Kurina et al. 2001). In MS, nearly half of newly diagnosed individuals report high levels of anxiety or depressive symptoms (Janssens et al. 2003). In RA, over one-third of individuals report high levels of emotional distress at diagnosis (Persson et al. 2005), and $80 \%$ of depressive disorders are diagnosed within 5 years (Wang et al. 2014). In a large Danish study using health claims data, several autoimmune diseases were associated with an increased risk of bipolar disorder within the first 4 years after diagnosis (thyrotoxicosis, MS, IBD, psoriasis, RA), or 5 or more years after diagnosis (autoimmune hepatitis) (Eaton et al. 2010). Similarly, several autoimmune diseases were associated with an increased risk of schizophrenia within the first 4 years after diagnosis (thyrotoxicosis, iridocyclitis, psoriasis, Sjogren's syndrome) or 5 or more years after diagnosis (thyrotoxicosis, Crohn's disease, autoimmune hepatitis). Collectively, this suggests the risk of developing psychiatric disorders is particularly high in the first 4 or 5 years post-diagnosis.

Several possibilities may explain the increased incidence of psychiatric disorders during the pre-diagnosis period for IMID. First, this could reflect a prodromal period for the IMID in which inflammation has developed sufficiently to increase the risk of psychiatric 
disorders but not to precipitate typical clinical manifestations of IMID. Emerging evidence suggests that the risk of depression, anxiety disorders and bipolar disorder is influenced by the inflammation and activation of cell-mediated immunity (Vieira et al. 2010; Berk et al. 2013; Rosenblat \& McIntyre, 2015). Second, psychiatric disorders and IMID may share common aetiologic factors. Some genetic loci are associated with the risk of both psychiatric and immune-mediated disorders (Wang et al. 2015). As well, environmental factors such as social adversity and chronic stress may induce biological changes which subsequently increase the risk of one or more chronic diseases (Cohen et al. 2012). In autoimmune disease, for example, common aetiologic factors with differing local pathogenetic effects may manifest as chronic immune disorders in one or more organ systems. Third, there may be a surveillance bias in the IMID cohorts, in which individuals begin to present to the health system for non-specific manifestations of IMID such as fatigue, thereby increasing their likelihood of obtaining diagnoses of other conditions. For example, a study of 5305 Americans with newly diagnosed MS found that $28.9 \%$ had seen a physician for fatigue, as based on billing codes, during the 3-year period before MS diagnosis; comparative data in individuals without MS were not available (Berger et al. 2013). Nonetheless, our point estimates were unchanged after adjusting for the annual number of physician visits. Fourth, the onset of psychiatric disorders could increase the subsequent risk of developing IMID. Depression is known to induce changes in immune function, including immunosuppression and inflammation (Nair et al. 1999; Yeragani et al. 2000; Irwin \& Miller, 2007), as well as hyperactivity of the hypothalamic pituitary axis (Stetler \& Miller, 2011). Individuals with generalised anxiety disorder may also have dysregulated T-cell function (Vieira et al. 2010). In addition, psychiatric disorders are associated with adverse health behaviours, while serious psychiatric disorders are associated with inequalities in health care provision that may lead to other health conditions (Lawrence \& Kisely, 2010). All of these aforementioned explanations largely consider the occurrence of psychiatric disorders in persons with IMID to be distinct comorbid conditions. However, the occurrence of psychiatric disorders pre-diagnosis of IMID could potentially be conceptualised as early symptoms of IMID rather than as distinct comorbid conditions. While this possibility would have important implications for the diagnostic evaluation and treatment of individuals with incident psychiatric disorders, our study does not allow us to distinguish these possibilities. Moreover, it is likely that psychiatric disorders are comorbid conditions in some persons with IMID but direct effects of IMID in other persons with IMID.
Future studies are necessary to evaluate all of the potential explanations for the association between psychiatric disorders and subsequent development of IMID. Such studies would benefit from measuring genetic factors, as well as environmental factors including infectious exposures, health behaviours and measures of social adversity in longitudinal population-based cohorts with and without psychiatric disorders. Further pathobiological study of mental health diseases in the context of IMID using brain imaging and serological and tissue biomarker studies will also be needed to discern the pathogenesis of psychiatric disorders in IMID (Najjar et al. 2013; Pearlman \& Najjar, 2014)

This study was limited to a single Canadian province potentially reducing generalisability. However, we have found in previous studies of MS that our findings regarding the relative risk of psychiatric comorbidity after MS diagnosis and temporal trends are consistent across other Canadian provinces (Marrie et al. 2015b). Administrative data are not collected for research, but we have validated our approaches to identifying IBD, MS, RA and psychiatric comorbidity using these data (Bernstein et al. 1999; Marrie et al. 2010; Marrie et al. 2013; Marrie et al. 2016). These data only capture individuals who have sought care through hospitals or physician visits and do not capture individuals with psychiatric disorders diagnosed by non-physician mental health providers, nor those who have not sought any treatment, although this would affect individuals with and without IMID. As administrative data lack clinical details, we could not evaluate associations between the timing of onset of psychiatric comorbidity and specific characteristics of the IMID, such as severity of illness.

We found that the incidence of psychiatric comorbidity is increased as early as 5-10 years before the diagnosis of IMID. Future studies should elucidate whether this reflects shared risk factors for psychiatric disorders and IMID, a shared final common inflammatory pathway or other aetiology. From a clinical perspective, the increased risk of psychiatric comorbidity at the time of IMID diagnosis and post-diagnosis highlights the clinical importance of detecting and treating these comorbidities in an appropriate, timely fashion. Detection will require clinical awareness and efficient screening strategies, while treatment will require effective collaborative mental health models. Our findings also raise questions regarding appropriate diagnostic investigations when individuals without an apparent history of IMID present with incident psychiatric disorders; future studies will need to address this issue.

\section{Supplementary material}

The supplementary material for this article can be found at https://doi.org/10.1017/S2045796017000579. 


\section{Acknowledgement}

The sponsors had no role in the design and conduct of the study; collection, management, analysis and interpretation of the data; and preparation, review or approval of the manuscript. The authors acknowledge the Manitoba Centre for Health Policy for use of the Manitoba Population Research Data Repository under project \#2014-030 (HIPC \#2015/2015-19A). The results and conclusions presented are those of the authors and no official endorsement by the Manitoba Centre for Health Policy, Manitoba Health or other data providers is intended or should be inferred. Members of the CIHR Team in Defining the Burden and Managing the Effects of Psychiatric Comorbidity in Chronic Immunoinflammatory Disease are: Ruth Ann Marrie, James M Bolton, Jitender Sareen, John R Walker, Scott B Patten, Alexander Singer, Lisa M. Lix, Carol A Hitchon, Renée El-Gabalawy, PhD, Alan Katz, John D Fisk, Charles N Bernstein, Lesley Graff, Lindsay Berrigan, Ryan Zarychanski, Christine Peschken, James Marriott.

\section{Financial support}

This study was funded by the Canadian Institutes of Health Research (THC-135234), Crohn's and Colitis Canada, and the Waugh Family Chair in Multiple Sclerosis (to RAM). Dr Bernstein is supported in part by the Bingham Chair in Gastroenterology. Dr Sareen is supported by CIHR \#333252. Dr Lix is supported by a Research Manitoba Chair.

\section{Conflict of Interest}

Ruth Ann Marrie has conducted clinical trials for Sanofi Aventis. Charles Bernstein has consulted to Abbvie Canada, Janssen Canada, Pfizer Canada, Shire Canada, Takeda Canada, and has received unrestricted educational grants from Abbvie Canada, Janssen Canada, Shire Canada and Takeda Canada. He has been on speaker's bureau of Abbvie Canada and Shire Canada. Jitender Sareen holds stock in Johnson and Johnson. All other authors have no conflicts of interest to declare.

\section{Ethical Standard}

The authors assert that all procedures contributing to this work comply with the ethical standards of the relevant national and institutional committees on human experimentation and with the Helsinki Declaration of 1975, as revised in 2008.

\section{Availability of Data and Materials}

The authors received permission to access the data used in this study; however, they are unable to share the data as they are not the data custodians.

\section{References}

Alamanos Y, Voulgari PV, Drosos AA (2006). Incidence and prevalence of rheumatoid arthritis, based on the 1987 American College of Rheumatology criteria: a systematic review. Seminars in Arthritis and Rheumatism 36, 182-188.

Ang DC, Choi H, Kroenke K, Wolfe F (2005). Comorbid depression is an independent risk factor for mortality in patients with rheumatoid arthritis. Journal of Rheumatology 32, 1013-1019.

Berger JR, Pocoski J, Preblick R, Boklage S (2013). Fatigue heralding multiple sclerosis. Multiple Sclerosis Journal 19, 1526-1532.

Berk M, Williams L, Jacka F, O'Neil A, Pasco J, Moylan S, Allen N, Stuart A, Hayley A, Byrne M, Maes M (2013). So depression is an inflammatory disease, but where does the inflammation come from? BMC Medicine 11, 200.

Bernstein CN, Blanchard JF, Rawsthorne P, Wajda A (1999). Epidemiology of Crohn's disease and ulcerative colitis in a central Canadian province: a population-based study. American Journal of Epidemiology 149, 916-924.

Bernstein CN, Longobardi T, Finlayson G, Blanchard JF (2012). Direct medical cost of managing IBD patients: a Canadian population-based study. Inflammatory Bowel Diseases 18, 1498-1508.

Byatt N, Rothschild AJ, Riskind P, Ionete C, Hunt AT (2011). Relationships between multiple sclerosis and depression. The Journal of Neuropsychiatry and Clinical Neurosciences 23, 198-200.

Canadian Institute for Health Information (2007). The Burden of Neurological Diseases, Disorders and Injuries in Canada. Ottawa.

Chateau D, Metge C, Prior H, Soodeen RA (2012). Learning from the census: the Socio-economic Factor Index (SEFI) and health outcomes in Manitoba. Canadian Journal of Public Health 103, S23-S27.

Chiu C-J., Hsu Y-C., Tseng S-P. (2016). Psychological prognosis after newly diagnosed chronic conditions: socio-demographic and clinical correlates. International Psychogeriatrics, 1-12.

Cohen S, Janicki-Deverts D, Doyle WJ, Miller GE, Frank E, Rabin BS, Turner RB (2012). Chronic stress, glucocorticoid receptor resistance, inflammation, and disease risk. Proceedings of the National Academy of Sciences of the United States of America 109, 5995-5999.

Eaton WW, Pedersen MG, Nielsen PR, Mortensen PB (2010). Autoimmune diseases, bipolar disorder, and non-affective psychosis. Bipolar Disorders 12, 638-646.

Faust AH, Halpern LF, Danoff-Burg S, Cross RK (2012). Psychosocial factors contributing to inflammatory bowel disease activity and health-related quality of life. Gastroenterology \& Hepatology (New York) 8, 173-181.

Hauser W, Janke KH, Klump B, Hinz A (2011). Anxiety and depression in patients with inflammatory bowel disease: comparisons with chronic liver disease patients and the general population. Inflammatory Bowel Disease 17, 621-632.

Health Canada (2003). Arthritis in Canada: An Ongoing Challenge. Health Canada: Ottawa.

Hitchon CA, Khan S, Elias B, El-Gabalawy HS, Katz A, Peschken CA (2014). First Nations persons have an 
increased risk of developing rheumatoid arthritis with an early onset age but are seen less frequently by rheumatologists: a population based study. In ACR/ARHP Annual Meeting. American College of Rheumatology: Boston, MA.

Hoang H, Laursen B, Stenager EN, Stenager E (2016). Psychiatric co-morbidity in multiple sclerosis: the risk of depression and anxiety before and after MS diagnosis. Multiple Sclerosis Journal 22, 347-353.

Irwin MR, Miller AH (2007). Depressive disorders and immunity: 20 years of progress and discovery. Brain, Behavior and Immunity 21, 374-383.

Janssens ACJW, van Doorn PA, de Boer JB, van der Meché FGA, Passchier J, Hintzen RQ (2003). Impact of recently diagnosed multiple sclerosis on quality of life, anxiety, depression and distress of patients and partners. Acta Neurologica Scandinavica 108, 389-395.

Kurina LM, Goldacre MJ, Yeates D, Gill LE (2001). Depression and anxiety in people with inflammatory bowel disease. Journal of Epidemiology and Community Health 55, 716-720.

Lawrence D, Kisely S (2010). Inequalities in healthcare provision for people with severe mental illness. Journal of Psychopharmacology 24, 61-68.

Logan I, Bowlus CL (2010). The geoepidemiology of autoimmune intestinal diseases. Autoimmunity Reviews 9, A372-A378.

Marrie RA, Yu N, Blanchard JF, Leung S, Elliott L (2010). The rising prevalence and changing age distribution of multiple sclerosis in Manitoba. Neurology 74, 465-471.

Marrie RA, Fisk JD, Yu BN, Leung S, Elliott L, Caetano P, Warren S, Evans C, Wolfson C, Svenson LW, Tremlett H, Blanchard JF, Patten SB (2013). Mental comorbidity and multiple sclerosis: validating administrative data to support population-based surveillance. BMC Neurology 13, 16.

Marrie RA, Elliott L, Marriott J, Cossoy M, Blanchard J, Leung S, Yu N (2015a). Effect of comorbidity on mortality in multiple sclerosis. Neurology 85, 240-247.

Marrie RA, Fisk JD, Tremlett H, Wolfson C, Warren S, Tennakoon A, Leung S, Patten SB (2015b). Differences in the burden of psychiatric comorbidity in MS vs the general population. Neurology 85, 1972-1979.

Marrie RA, Walker JR, Graff LA, Lix LM, Bolton JM, Nugent Z, Targownik LE, Bernstein CN (2016). Performance of administrative case definitions for depression and anxiety in inflammatory bowel disease. Journal of Psychosomatic Research 89, 107-113.

Matcham F, Rayner L, Steer S, Hotopf M (2013). The prevalence of depression in rheumatoid arthritis: a systematic review and meta-analysis. Rheumatology 52, 2136-2148.

Mitchell AJ, Benito-León J, González J-MM, Rivera-Navarro J (2005). Quality of life and its assessment in multiple sclerosis: integrating physical and psychological components of wellbeing. The Lancet Neurology 4, 556-566.

Mok C, Lok E, Cheung E (2012). Concurrent psychiatric disorders are associated with significantly poorer quality of life in patients with rheumatoid arthritis. Scandinavian Journal of Rheumatology 41, 253-259.
Nair GV, Gurbel PA, O'Connor CM, Gattis WA, Murugesan SR, Serebruany VL (1999). Depression, coronary events, platelet inhibition, and serotonin reuptake inhibitors. American Journal of Cardiology 84, 321-323.

Najjar S, Pearlman DM, Alper K, Najjar A, Devinsky O (2013). Neuroinflammation and psychiatric illness. Journal of Neuroinflammation 10, 43.

Pearlman DM, Najjar S (2014). Meta-analysis of the association between $\mathrm{N}$-methyl-d-aspartate receptor antibodies and schizophrenia, schizoaffective disorder, bipolar disorder, and major depressive disorder. Schizophrenia Research 157, 249-258.

Persson LO, Larsson BM, Nived K, Eberhardt K (2005). The development of emotional distress in 158 patients with recently diagnosed rheumatoid arthritis: a prospective 5-year follow-up study. Scandinavian Journal of Rheumatology 34, 191-197.

Pugliatti M, Sotgiu S, Rosati G (2002). The worldwide prevalence of multiple sclerosis. Clinical Neurology and Neurosurgery 104, 182-191.

Rosenblat JD, McIntyre RS (2015). Are medical comorbid conditions of bipolar disorder due to immune dysfunction? Acta Psychiatrica Scandinavica 132, 180-191.

Rupp I, Boshuizen HC, Jacobi CE, Dinant HJ, van den Bos G (2004). Comorbidity in patients with rheumatoid arthritis: effect on health-related quality of life. The Journal of Rheumatology 31, 58-65.

Stetler C, Miller GE (2011). Depression and hypothalamic-pituitary-adrenal activation: a quantitative summary of four decades of research. Psychosomatic Medicine 73, 114-126.

Vieira MMM, Ferreira TB, Pacheco PAF, Barros PO, Almeida CRM, Araújo-Lima CF, Silva-Filho RG, Hygino J, Andrade RM, Linhares UC, Andrade AFB, Bento CAM (2010). Enhanced Th17 phenotype in individuals with generalized anxiety disorder. Journal of Neuroimmunology 229, 212-218.

Walker JR, Ediger JP, Graff LA, Greenfeld JM, Clara I, Lix L, Rawsthorne P, Miller N, Rogala L, McPhail CM, Bernstein CN (2008). The Manitoba IBD cohort study: a population-based study of the prevalence of lifetime and 12-month anxiety and mood disorders. American Journal of Gastroenterology 103, 1989-1997.

Wang Q, Yang C, Gelernter J, Zhao H (2015). Pervasive pleiotropy between psychiatric disorders and immune disorders revealed by integrative analysis of multiple GWAS. Human Genetics 134, 1195-1209.

Wang S-L, Chang C-H, Hu L-Y, Tsai S-J, Yang AC, You Z-H (2014). Risk of developing depressive disorders following rheumatoid arthritis: a nationwide population-based study. PLOS ONE 9, e107791.

WHO Collaborating Centre for Drug Statistics Methodology (2012). Guidelines for ATC Classification and DDD Assignment 2013. Oslo.

Yeragani VK, Pohl R, Jampala VC, Balon R, Ramesh C, Srinivasan K (2000). Increased QT variability in patients with panic disorder and depression. Psychiatry Research 93, 225-235. 